\title{
POLYNOMIAL SPLINE COLLOCATION METHODS FOR SECOND-ORDER VOLTERRA INTEGRODIFFERENTIAL EQUATIONS
}

\author{
EDRIS RAWASHDEH, DAVID MCDOWELL, and LEELA RAKESH
}

Received 21 October 2002

We have presented a method for the construction of an approximation to the initial-value second-order Volterra integrodifferential equation (VIDE). The polynomial spline collocation methods described here give a superconvergence to the solution of the equation.

2000 Mathematics Subject Classification: 34Bxx, 45L10, 65D05, 65D15.

1. Introduction. In order to discuss the numerical solution of the second-order VIDEs we consider the following linear integrodifferential equation:

$$
y^{(2)}(t)=q(t)+\sum_{i=0}^{1} p_{i}(t) y^{(i)}(t)+\sum_{i=0}^{1} \int_{0}^{t} k_{i}(t, s) y^{(i)}(s) d s, \quad t \in I:=[0, T],
$$

with

$$
y(0)=y_{0}, \quad y^{(1)}(0)=y_{1},
$$

where $q: I \rightarrow R, p_{i}: I \rightarrow R$, and $k_{i}: D \rightarrow R(i=0,1)$ (with $D:=\{(t, s): 0 \leq s \leq t \leq$ $T\}$ ) are given functions and are assumed to be (at least) continuous in the respective domains. For more details of these equations, many other interesting methods for the approximated solution and stability procedures are available in earlier literatures [1, $3,4,5,6,7,8,11]$. The above equation is usually known as basis test equation and is suggested by Brunner and Lambert [5]. Since then it has been widely used for analyzing the solution and stability properties of various methods.

Second-order VIDEs of the above form (1.1) will be solved numerically using polynomial spline spaces. In order to describe these approximating polynomial spline spaces, let $\prod_{N}: 0=t_{0}<t_{1}<\cdots<t_{N}=T$ be the mesh for the interval $I$, and set

$$
\begin{aligned}
\sigma_{n} & :=\left[t_{n}, t_{n+1}\right], \quad h_{n}:=t_{n+1}-t_{n}, \quad n=0,1, \ldots, N-1, \\
h & =\max \left\{h_{n}: 0 \leq n \leq N-1\right\} \quad \text { (mesh diameter), } \\
Z_{N} & :=\left\{t_{n}: n=1,2, \ldots, N-1\right\}, \quad \bar{Z}_{N}=Z_{N} \cup\{T\} .
\end{aligned}
$$

Let $\pi_{m+d}$ be the set of (real) polynomials of degree not exceeding $m+d$, where $m \geq 1$ and $d \geq-1$ are given integers. The solution $y$ to the initial-value problem (1.1), (1.2) 
will be approximated by an element $u$ in the polynomial spline space,

$$
\begin{gathered}
S_{m+d}^{(d)}\left(Z_{N}\right):=\left\{u:=\left.u(t)\right|_{t \in \sigma_{n}}:=u_{n}(t) \in \pi_{m+d}, n=0,1, \ldots, N-1,\right. \\
\left.u_{n-1}^{(j)}\left(t_{n}\right)=u_{n}^{(j)}\left(t_{n}\right) \text { for } j=0,1, \ldots, d, t_{n} \in Z_{N}\right\},
\end{gathered}
$$

that is, by a polynomial spline function of degree $m+d$ which possesses the knots $Z_{N}$ and is $d$ times continuously differentiable on $I$. If $d=-1$, then the elements of $S_{m-1}^{(-1)}\left(Z_{N}\right)$ may have jump discontinuities at the knots $Z_{N}$. Initial value problems, such as (1.1) and (1.2), have often been solved by collocation method in polynomial spines spaces $S_{m}^{(0)}\left(Z_{N}\right)$ and $S_{m}^{(1)}\left(Z_{N}\right)$.

According to M. Miculá and G. Micula [10], an element $u \in S_{m+d}^{(d)}\left(Z_{N}\right)$ has the following form: for all $n=0,1, \ldots, N-1$ and $t \in \sigma_{n}$,

$$
u(t)=u_{n}(t)=\sum_{r=0}^{d} \frac{u_{n-1}^{(r)}\left(t_{n}\right)}{r !}\left(t-t_{n}\right)^{r}+\sum_{r=1}^{m} a_{n, r}\left(t-t_{n}\right)^{d+r}
$$

where

$$
u_{-1}^{r}(0):=\left[\frac{d^{r}}{d t^{r}} u(t)\right]_{t=0}=y^{(r)}(0), \quad r=0,1, \ldots, d
$$

From (1.5), we see that the element $u \in S_{m+d}^{(d)}\left(Z_{N}\right)$ is well defined provided the coefficients $\left\{a_{n, r}\right\}_{r=1, \ldots, m}$ are known. In order to determine these coefficients, we consider a set of collocation parameters $\left\{c_{j}\right\}_{j=1, \ldots, m}$, where $0<c_{1}<\cdots<c_{m} \leq 1$, and define the set of collocation points as

$$
X(N):=\bigcup_{n=0}^{N-1} X_{n}, \quad \text { with } X_{n}:=\left\{t_{n, j}:=t_{n}+c_{j} h_{n}, j=1,2, \ldots, m\right\}
$$

The approximate solution $u \in S_{m+d}^{(d)}\left(Z_{N}\right)$ will be determined by imposing the condition that $u$ satisfies the following initial-value problem on $X(N)$ :

$$
u^{(2)}(t)=q(t)+\sum_{i=0}^{1} p_{i}(t) u^{(i)}(t)+\sum_{i=0}^{1} \int_{0}^{t} k_{i}(t, s) u^{(i)}(s) d s, \quad \forall t \in X(N),
$$

with

$$
u(0)=y_{0}, \quad u^{(1)}(0)=y_{1},
$$

with a uniform the mesh sequence $\left\{\prod_{N}\right\}, h_{n}=h$, for all $n=0,1, \ldots, N-1$, but for small $h,(1.8)$ has a unique solution $\left\{a_{n, j}\right\}_{j=1, \ldots, m}$, for all $n=0,1, \ldots, N-1$. 
Therefore, the modified collocation equation (1.8) can be rewritten as

$$
\begin{aligned}
u_{n}^{\prime \prime}\left(t_{n, j}\right)= & q\left(t_{n, j}\right)+p_{0}\left(t_{n, j}\right) u_{n}\left(t_{n, j}\right)+p_{1}\left(t_{n, j}\right) u_{n}^{\prime}\left(t_{n, j}\right) \\
& +h_{n} \phi_{n, n}^{j}\left[u_{n}\right]+\sum_{i=0}^{n-1} h_{i} \phi_{n, i}^{j}\left[u_{i}\right] \\
& +\psi_{n, n}^{j}\left[u_{n}^{\prime}\right]+\sum_{i=0}^{n-1} \psi_{n, i}^{j}\left[u_{i}^{\prime}\right], \quad j=1, \ldots, m, n=0,1, \ldots, N-1,
\end{aligned}
$$

where

$$
\begin{aligned}
& \phi_{n, i}^{j}\left[u_{i}\right]= \begin{cases}\int_{0}^{c_{j}} k_{0}\left(t_{n, j}, t_{n}+v h_{n}\right) u_{n}\left(t_{n}+v h_{n}\right) d v, & \text { if } i=n, \\
\int_{0}^{1} k_{0}\left(t_{n, j}, t_{i}+v h_{i}\right) u_{i}\left(t_{i}+v h_{i}\right) d v, & \text { if } i=0,1, \ldots, n-1,\end{cases} \\
& \psi_{n, i}^{j}\left[u_{i}\right]= \begin{cases}\int_{0}^{c_{j}} k_{1}\left(t_{n, j}, t_{n}+v h_{n}\right) u_{n}^{\prime}\left(t_{n}+v h_{n}\right) d v, & \text { if } i=n, \\
\int_{0}^{1} k_{1}\left(t_{n, j}, t_{i}+v h_{i}\right) u_{i}^{\prime}\left(t_{i}+v h_{i}\right) d v, & \text { if } i=0,1, \ldots, n-1 .\end{cases}
\end{aligned}
$$

In most applications the integrals appearing in (1.11) cannot be evaluated analytically, so we seek suitable quadrature formulas as follows:

$$
\begin{aligned}
& \hat{\phi}_{n, i}^{j}\left[u_{i}\right]= \begin{cases}\sum_{l=1}^{\mu_{1}} w_{j, l} k_{0}\left(t_{n, j}, t_{n}+d_{j, l} h_{n}\right) u_{n}\left(t_{n}+d_{j, l} h_{n}\right), & \text { if } i=n, \\
\sum_{l=1}^{\mu_{0}} w_{l} k_{0}\left(t_{n, j}, t_{i}+d_{l} h_{i}\right) u_{i}\left(t_{i}+d_{l} h_{i}\right), & \text { if } i=0,1, \ldots, n-1,\end{cases} \\
& \hat{\psi}_{n, i}^{j}\left[u_{i}^{\prime}\right]= \begin{cases}\sum_{l=1}^{\mu_{1}} w_{j, l} k_{1}\left(t_{n, j}, t_{n}+d_{j, l} h_{n}\right) u_{n}^{\prime}\left(t_{n}+d_{j, l} h_{n}\right), & \text { if } i=n, \\
\sum_{l=1}^{\mu_{0}} w_{l} k_{1}\left(t_{n, j}, t_{i}+d_{l} h_{i}\right) u_{i}^{\prime}\left(t_{i}+d_{l} h_{i}\right), & \text { if } i=0,1, \ldots, n-1,\end{cases}
\end{aligned}
$$

where $\mu_{0}$ and $\mu_{1}$ are two given positive integers, $\left\{d_{l}\right\}$ and $\left\{d_{j, l}\right\}$ are two sets of parameters satisfying the following conditions:

$$
0 \leq d_{1}<\cdots<d_{\mu_{0}} \leq 1, \quad 0 \leq d_{j, 1}<\cdots<d_{j, \mu_{1}} \leq c_{j}, \quad(j=1, \ldots, m),
$$

and $w_{l}, w_{j, l}$ denote the quadrature weights.

The corresponding quadrature error terms are defined as

$$
\begin{aligned}
E_{n, i}^{j}\left[u_{i}\right] & =\phi_{n, i}^{j}\left[u_{i}\right]-\hat{\phi}_{n, i}^{j}\left[u_{i}\right], \\
E_{n, i}^{j}\left[u_{i}^{\prime}\right] & =\psi_{n, i}^{j}\left[u_{i}^{\prime}\right]-\hat{\psi}_{n, i}^{j}\left[u_{i}^{\prime}\right], \\
j=1,2, \ldots, m, \quad i & =0,1, \ldots, n \quad(n=0,1, \ldots, N-1) .
\end{aligned}
$$


By the above quadrature process (1.10) is a fully discretized collocation method which defines an element $\hat{u} \in S_{m+d}^{(d)}\left(Z_{N}\right)$ (which, in general, will be different from the approximate solution $u$ determined by the exact collocation equation (1.10)). The full discretized version of the collocation equation (1.10) is

$$
\begin{aligned}
\hat{u}_{n}^{\prime \prime}\left(t_{n, j}\right)= & q\left(t_{n, j}\right)+p_{0}\left(t_{n, j}\right) \hat{u}_{n}\left(t_{n, j}\right)+p_{1}\left(t_{n, j}\right) \hat{u}_{n}^{\prime}\left(t_{n, j}\right) \\
& +h_{n} \hat{\phi}_{n, n}^{j}\left[\hat{u}_{n}\right]+\sum_{i=0}^{n-1} h_{i} \hat{\phi}_{n, i}^{j}\left[\hat{u}_{i}\right] \\
& +\hat{\psi}_{n, n}^{j}\left[\hat{u}_{n}^{\prime}\right]+\sum_{i=0}^{n-1} \hat{\psi}_{n, i}^{j}\left[\hat{u}_{i}^{\prime}\right], \quad j=1, \ldots, m, n=0,1, \ldots, N-1 .
\end{aligned}
$$

Similarly, the approximate discretized solution $\hat{u} \in S_{m+d}^{(d)}\left(Z_{N}\right)$ has the following form:

$$
\hat{u}(t)=\hat{u}_{n}(t)=\sum_{r=0}^{d} \frac{\hat{u}_{n-1}^{(r)}\left(t_{n}\right)}{r !}\left(t-t_{n}\right)^{r}+\sum_{r=1}^{m} \hat{a}_{n, r}\left(t-t_{n}\right)^{d+r},
$$

with the assumption that

$$
\hat{u}_{-1}^{(r)}(0):=y^{(r)}(0), \quad r=0,1, \ldots, d
$$

The collocation equations (1.10) and (1.16) represent recursive systems, for each $n=$ $0,1, \ldots, N-1$, which yield the coefficients $\left\{a_{n, r}\right\}_{r=1, \ldots, m}$ and $\left\{\hat{a}_{n, r}\right\}_{r=1, \ldots, m}$, respectively. Once the coefficients are known, the values of $u, \hat{u}$ together with their derivatives are determined on $\sigma_{n}$ by (1.5) and (1.17), respectively.

On each of the $N$ subintervals of $I$ we have to solve an $m \times m$ system of linear equations. On the first subinterval $\sigma_{0}, d+1$ additional equations are furnished by the $d+1$ initial conditions (1.6) and (1.18).

2. Global convergence criteria. If the given functions $q, p_{i}$, and $k_{i}(i=0,1)$ are of class $m+d$ on their domain of definition, then the VIDE (1.1) has a unique solution $y$, which is of class $m+d+2$. Let $\varphi_{n}$ be the restriction of the function $\varphi$ on $I$ in the subinterval $\sigma_{n}$, for $n=0,1, \ldots, N-1$, and the norm is defined by

$$
\|\varphi\|_{\infty}=\sup \left\{\left|\varphi_{n}(t)\right|: t \in \sigma_{n}, n=0,1, \ldots, N-1\right\}
$$

The following theorem describes the order of global convergence of $u \in S_{m+d}^{(d)}\left(Z_{N}\right)$, $d \in\{0,1,2\}$.

THEOREM 2.1. Let $q, p_{i}$, and $k_{i}$ be $m+d$ times continuously differentiable in the respective domains $I$ and $S$. For $d \in\{0,1,2\}$, every choice of collocation parameters $\left\{c_{j}\right\}_{j=1, m}$, with $0<c_{1}<\cdots<c_{m} \leq 1$, and all quasiuniform mesh sequences $\left\{\prod_{N}\right\}$ with sufficiently small $h>0$, the following hold: 
(i) the exact collocation equation (1.10) defines a unique approximation $u \in S_{m+d}^{(d)}\left(Z_{N}\right)$ and the resulting error function $e:=y-u$ satisfies

$$
\left\|e^{(k)}\right\|_{\infty} \leq C_{k} h^{m+d+1-k}, \quad \forall k=0,1, \ldots, m+d,
$$

where $C_{k}$ 's are finite constants independent of $h$;

(ii) if the quadrature formulas (1.12) and (1.13) satisfy that for $i=0,1, \ldots, n-1$,

$$
\begin{aligned}
& \int_{0}^{1} \phi\left(t_{i}+\tau h_{i}\right) d \tau-\sum_{l=1}^{\mu_{1}} w_{l} \phi\left(t_{i}+d_{i} h_{i}\right)=O\left(h_{i}^{\gamma_{1}}\right), \\
& \int_{0}^{1} \psi\left(t_{i}+\tau h_{i}\right) d \tau-\sum_{l=1}^{\mu_{1}} w_{l} \psi\left(t_{i}+d_{i} h_{i}\right)=O\left(h_{i}^{\gamma_{1}}\right),
\end{aligned}
$$

and for $j=1, \ldots, m$,

$$
\begin{aligned}
& \int_{0}^{c_{j}} \phi\left(t_{n}+\tau h_{n}\right) d \tau-\sum_{l=1}^{\mu_{0}} w_{j, l} \phi\left(t_{n}+d_{j, l} h_{n}\right)=O\left(h_{n}^{r_{0}}\right), \\
& \int_{0}^{c_{j}} \psi\left(t_{n}+\tau h_{n}\right) d \tau-\sum_{l=1}^{\mu_{0}} w_{j, l} \psi\left(t_{n}+d_{j, l} h_{n}\right)=O\left(h_{n}^{r_{0}}\right),
\end{aligned}
$$

whenever the integrand is sufficiently smooth, then for all the discretized approximation solution $\hat{u} \in S_{m+d}^{(d)}\left(Z_{N}\right)$ defined by (1.17), the following relationships hold:

$$
\begin{array}{ll}
\left\|\varepsilon^{(k)}\right\|_{\infty}:=\left\|u^{(k)}-\hat{u}^{(k)}\right\|_{\infty} \leq Q_{k} h^{s_{0}-k}, & \text { for } k=0,1, \ldots, s_{0}, \\
\left\|\hat{e}^{(k)}\right\|_{\infty}:=\left\|y^{(k)}-\hat{u}^{(k)}\right\|_{\infty} \leq \hat{C}_{k} h^{s_{1}-k}, & \text { for } k=0,1, \ldots, s_{1},
\end{array}
$$

where $s_{0}=\min \left\{r_{0}+1, r_{1}\right\}+1, s_{1}=\min \left\{s_{0}, m+d+1\right\}$, and $Q_{k}, \hat{C}_{k}$ are finite constants independent of $h$.

Proof. We will prove the theorem by induction using the same technique as in $[2,3,9]$.

(i) For $n=0,1, \ldots, N-1$ and for all $t=t_{n}+\tau h_{n} \in \sigma_{n}(\tau \in(0,1])$, the exact solution $y$ can be obtained by Taylor series expansion:

$$
y\left(t_{n}+\tau h_{n}\right)=\sum_{r=0}^{m+d} \frac{y^{(r)}\left(t_{n}\right) \tau^{r}}{r !} h_{n}^{r}+h_{n}^{m+d+1} R_{n}(\tau),
$$

where

$$
R_{n}(\tau)=\frac{1}{(m+d) !} \int_{0}^{\tau} y^{(m+d+1)}\left(t_{n}+\eta h_{n}\right)(\tau-\eta)^{m+d} d \eta
$$

If the restriction of $u \in S_{m+d}^{(d)}\left(Z_{N}\right)$ to the subinterval $\sigma_{n}$ is given by (1.5), then by using (2.9), the error function on this subinterval has the form

$$
e_{n}\left(t_{n}+\tau h_{n}\right)=\sum_{r=0}^{d} \frac{e_{n-1}^{(r)}\left(t_{n}\right)}{r !} \tau^{r} h_{n}^{r}+h_{n}^{p} \sum_{r=1}^{m} \beta_{n, r} \tau^{d+r}+h_{n}^{m+d+1} R_{n}(\tau),
$$


where

$$
h_{n}^{p} \beta_{n, r}:=\left(\frac{y^{(d+r)}\left(t_{n}\right)-a_{n, r}}{(d+r) !}\right) h_{n}^{d+r} .
$$

Subtracting the collocation equation (1.8) from the integrodifferential equation (1.1), replacing $t$ by $t_{n, j}$, using (1.6) for $n=0$, and utilizing the expression (2.11) for $e_{n}$ yields

$$
\begin{gathered}
h_{0}^{p-2} D_{0} \beta_{0}=h_{0}^{m+d-1} r_{0}, \text { for } n=0, \\
h_{n}^{p-2} D_{n} \beta_{n}=F_{n} E_{n}+h_{n}^{m+d-1} r_{n}+\sum_{i=1}^{n-1}\left(h_{i} q_{n, i}+p_{n, i}\right), \text { for } n>0,
\end{gathered}
$$

where $D_{n}$ is an $m \times m$ matrix, $F_{n}$ is an $m \times(d+1)$ matrix, $E_{n}$ is a $(d+1)$ vector, and $\beta_{n}, r_{n}, q_{n, i}, p_{n, i}$ are $m$ vectors whose elements are given by

$$
\begin{aligned}
& \left(D_{n}\right)_{j, r}=c_{j}^{d+r-2}\left[(d+r)(d+r-1)-h_{n}^{2} p_{0}\left(t_{n}+d_{j} h_{n}\right) c_{j}^{2}-(d+r) c_{j} h_{n} p_{1}\left(t_{n}+c_{j} h_{n}\right)\right] \\
& -h_{n}^{3} \int_{0}^{c_{j}} k_{0}\left(t_{n, j}, t_{n}+\tau h_{n}\right) \tau^{d+r} d \tau \\
& -(d+r) h_{n}^{2} \int_{0}^{c_{j}} k_{1}\left(t_{n, j}, t_{n}+\tau h_{n}\right) \tau^{d+r-1} d \tau, \\
& \left(F_{n}\right)_{j, r}= \begin{cases}p_{0}\left(t_{n, j}\right)+h_{n} \int_{0}^{c_{j}} k_{0}\left(t_{n, j}, t_{n}+\tau h_{n}\right) d \tau, & \text { if } r=0, \\
h_{n} c_{j} p_{0}\left(t_{n, j}\right) h_{n}+p_{1}\left(t_{n, j}\right)+h_{n}^{2} \int_{0}^{c_{j}} k_{0}\left(t_{n, j}, t_{n}+\tau h_{n}\right) \tau d \tau & \\
\quad+h_{n} \int_{0}^{c_{j}} k_{1}\left(t_{n, j}, t_{n}+\tau h_{n}\right) d \tau, & \text { if } r=1, \\
\frac{h_{n}^{r-2}\left[-r(r-1) c_{j}^{r-2}+h_{n}^{2} c_{j}^{r} p_{0}\left(t_{n, j}\right)+r h_{n} c_{j}^{r-1} p_{1}\left(t_{n, j}\right)\right]}{r !}[ \\
+h_{n}^{3} \int_{0}^{c_{j}} k_{0}\left(t_{n, j}, t_{n}+\tau h_{n}\right) \tau^{r} d \tau & \\
+r h_{n}^{2} \int_{0}^{c_{j}} k_{1}\left(t_{n, j}, t_{n}+\tau h_{n}\right) \tau^{r-1} d \tau, & \text { if } r \geq 2,\end{cases} \\
& \left(E_{n}\right)_{r}=e_{n-1}^{(r)}\left(t_{n}\right) \\
& \left(\beta_{n}\right)_{r}=\beta_{n, r} \text { as defined in (2.12), } \\
& \left(r_{n}\right)_{j}=-R_{n}^{\prime \prime}\left(c_{j}\right)+h_{n}^{2} p_{0}\left(t_{n, j}\right) R_{n}\left(c_{j}\right)+h_{n} p_{1}\left(t_{n, j}\right) R_{n}^{\prime}\left(c_{j}\right) \\
& +h_{n}^{3} \int_{0}^{c_{j}} k_{0}\left(t_{n, j}, t_{n}+\tau h_{n}\right) R_{n}(\tau) d \tau \\
& +h_{n}^{2} \int_{0}^{c_{j}} k_{1}\left(t_{n, j}, t_{n}+\tau h_{n}\right) R_{n}^{\prime}(\tau) d \tau, \\
& \left(q_{n, i}\right)_{j}=\int_{0}^{c_{j}} k_{0}\left(t_{n, j}, t_{i}+\tau h_{i}\right) e_{i}\left(t_{i}+\tau h_{i}\right) d \tau, \\
& \left(p_{n, i}\right)_{j}=\int_{0}^{c_{j}} k_{1}\left(t_{n, j}, t_{i}+\tau h_{i}\right) e_{i}^{\prime}\left(t_{i}+\tau h_{i}\right) d \tau .
\end{aligned}
$$


Note that the matrix $D_{n}$ defined by the coefficient of $\left\{\beta_{n, j}\right\}$ on the left-hand side of (2.13) and (2.14) is invertible whenever $h_{n}$ is sufficiently small. This follows from the assumptions of the theorem and the fact that for $h_{n}=0$ the determinant of this matrix is essentially a Vandermonde determinant. Therefore, for sufficiently small $h_{n}>0$, the matrix $D_{n}$ possesses a uniformly bounded inverse.

By the hypothesis on $p_{i}$ and $k_{i}$, the $\ell_{1}$-norm of the vector $r_{0}$ is uniformly bounded. Hence, for $p=m+d+1$, (2.13) leads to

$$
\left\|\beta_{0}\right\|_{1}:=\sum_{l=1}^{m}\left|\beta_{0, l}\right| \leq\left\|D_{0}^{-1}|||| r_{0}\right\|=: M_{0} .
$$

From (2.11) we have the following result:

$$
\begin{aligned}
\left|e_{0}\left(t_{0}+\tau h_{0}\right)\right| & \leq h^{m+d+1}\left(M_{0}+\left|R_{0}(\tau)\right|\right) \\
& \leq C_{0} h^{m+d+1}, \quad \text { for } \tau \in(0,1] .
\end{aligned}
$$

By differentiating (2.11) $k$ times $(k=1,2, \ldots, m+d)$ and using (2.16), we obtain

$$
\left|e_{0}^{k}\left(t_{0}+\tau h_{0}\right)\right| \leq C_{0}^{k} h^{m+d+1-k}, \quad \text { for } \tau \in(0,1] .
$$

Suppose, for $j=0,1, \ldots, n-1$,

$$
\left|e_{j}^{k}\left(t_{j}+\tau h_{j}\right)\right| \leq C_{j}^{k} h^{m+d+1-k}, \quad \text { for } \tau \in(0,1], k=0,1, \ldots, m+d ;
$$

we prove that (2.19) holds for $j=n$. By the assumption of the theorem on $q, p_{i}$, and $k_{i}$ and (2.19), it follows that for sufficiently small $h,\left\|E_{n}\right\|_{1}=O\left(h^{m+1}\right),\left\|q_{n, i}\right\|_{1}=$ $O\left(h^{m+d+1}\right),\left\|p_{n, i}\right\|_{1}=O\left(h^{m+d}\right)(i=0,1, \ldots, n-1)$, and $\left\|r_{n}\right\|_{1}$ is bounded. Thus, for $p=m+d+1$ and $d \in\{0,1,2\},(2.14)$ leads to

$$
\left\|\beta_{n}\right\|_{1}:=\sum_{l=1}^{m}\left|\beta_{n, l}\right| \leq M_{n}+M_{n}^{\prime} h .
$$

Then, from (2.20) and (2.11), it follows that

$$
\left|e_{n}^{k}\left(t_{n}+\tau h_{n}\right)\right| \leq C_{n}^{k} h^{m+d+1-k}, \quad \text { for } \tau \in(0,1], k=0,1, \ldots, m+d .
$$

This completes the first assertion of the theorem.

(ii) By (1.5) and (1.17) for every $n=0,1, \ldots, N-1$, the error function $\varepsilon:=u-\hat{u}$ can be written as

$$
\varepsilon_{n}\left(t_{n}+\tau h_{n}\right)=\sum_{r=0}^{d} \frac{\varepsilon_{n-1}^{(r)}\left(t_{n}\right)}{r !} \tau^{r} h_{n}^{r}+h_{n}^{s_{0}} \sum_{r=1}^{m} \eta_{n, r} \tau^{d+r},
$$

where

$$
h_{n}^{s_{0}} \eta_{n, r}:=\left(a_{n, r}-\hat{a}_{n, r}\right) h_{n}^{d+r}
$$


By subtracting the discretized equation (1.16) from the exact collocation equation (1.10) and using (1.15) and (2.22), we have the following systems:

$$
\begin{gathered}
h_{0}^{s_{0}-2} \hat{D}_{0} \eta_{0}=h_{0} r_{0,0}+\bar{r}_{0,0}, \quad \text { for } n=0, \\
h_{n}^{s_{0}-2} \hat{D}_{n} \eta_{n}=\hat{F}_{n} \xi_{n}+h_{n} r_{n, n}+\sum_{i=1}^{n-1} h_{i} r_{n, i}+\bar{r}_{n, n}+\sum_{i=1}^{n-1} \bar{r}_{n, i}, \quad \text { for } n>0,
\end{gathered}
$$

where $r_{n, i}:=\left(E_{n, i}^{1}\left[u_{i}\right], \ldots, E_{n, i}^{m}\left[u_{i}\right]\right)^{T}$ and $\bar{r}_{n, i}:=\left(E_{n, i}^{1}\left[u_{i}^{\prime}\right], \ldots, E_{n, i}^{m}\left[u_{i}^{\prime}\right]\right)^{T}$. The matrices $\hat{D}_{n}, \hat{F}_{n}, \xi_{n}$ have the same orders as $D_{n}, F_{n}, E_{n}$ from (2.13) and (2.14). The difference between them is that the integrals are replaced with quadrature formulas (1.12) and (1.13).

The expressions (2.24) and (2.25) have the same structure as (2.13) and (2.14), respectively. The smoothness hypothesis and the assumption on the order of the quadrature formulas (2.3), (2.4), (2.5), and (2.6) imply $\left\|r_{n, n}\right\|_{1}=\left\|\bar{r}_{n, n}\right\|_{1}=O\left(h_{n}^{r_{0}}\right)$ and $\left\|r_{n, i}\right\|_{1}=$ $\left\|\bar{r}_{n, i}\right\|_{1}=O\left(h_{n}^{r_{1}}\right)$. Therefore, by the same reasoning in the proof of assertion (i), inequality (2.7) is true.

From (2.2) and (2.7),

$$
\left\|\hat{e}^{(k)}\right\|_{\infty}:=\left\|y^{(k)}-\hat{u}^{(k)}\right\|_{\infty} \leq\left\|e^{(k)}\right\|_{\infty}+\left\|\varepsilon^{(k)}\right\|_{\infty} \leq \hat{C}_{k} h^{s_{1}-k}
$$

for all $k=0,1, \ldots, s_{1}$, with $s_{1}=\min \left\{s_{0}, m+d+1\right\}$.

COROLLARY 2.2. Let the assumptions of Theorem 2.1 hold. If the quadrature formulas (1.12) and (1.13) are of interpolatory type, with $\mu_{0}=\mu_{1}=m+d$, then the approximation $\hat{u} \in S_{m+d}^{(d)}\left(Z_{n}\right)$ defined by the discretized collocation equation (1.16) leads to an error $\hat{e}(t)$ satisfying

$$
\left\|\hat{e}^{(k)}\right\|_{\infty}:=O\left(h^{m+d+1-k}\right),
$$

for $k=0, \ldots, m+d$, every choice of collocation parameters $\left\{c_{j}\right\}_{j=1, \ldots, m}$, with $0<c_{1}<$ $\cdots<c_{m} \leq 1$, and all quasiuniform mesh sequences $\left\{\prod_{N}\right\}$ with sufficiently small $h>0$, and $d \in\{0,1,2\}$.

If we use $\mu_{0}=\mu_{1}=m, d_{j}=c_{j}$, and $d_{j, l}=c_{j} c_{l}(j, l=1,2, \ldots, m)$ in the quadrature formulas, then our method leads to some simplifications. These simplifications are useful when they do not affect the convergence order given by Theorem 2.1, namely, $s_{1}=m+d+1$.

Corollary 2.3. If in the $\operatorname{VIDE}(1.1), q \in C^{m+d}(I), p_{i} \in C^{m+d}(I), k_{i} \in C^{m+d}(S)$, and $m \geq d$, then there exists the set of collocation parameters $\left\{c_{j}\right\}_{j=1, \ldots, m}$ such that for the approximation $\hat{u} \in S_{m+d}^{(d)}\left(Z_{n}\right)$ defined by the discretized collocation equation (1.16), where $\mu_{0}=\mu_{1}=m, d_{l}=c_{l}$, and $d_{j, l}=c_{j} c_{l}$,

$$
\left\|\hat{e}^{(k)}\right\|_{\infty}:=O\left(h^{m+d+1-k}\right),
$$

for $k=0, \ldots, m+d, d \in\{0,1,2\}$. 
Proof. If $\mu_{0}=\mu_{1}=m, m \geq d$, then we can choose the collocation parameters to be the zeros of the shifted Legendre polynomial $P_{m}(2 s-1)$ (i.e., the Gauss points for $(0,1)$, as the collocation parameters $\left.\left\{c_{j}\right\}_{j=1, \ldots, m}\right)$. Then in the quadrature formulas (2.3), (2.4), (2.5), and (2.6), $r_{0}=r_{1}=2 m$. Therefore, $s_{0}=2 m+1$ and $s_{1}=m+d+1$ in (2.7) and (2.8), respectively, which proves the corollary.

3. Local superconvergence on $\bar{Z}_{N}$. In many applications one is especially interested in obtaining higher-order approximations at the mesh points $\bar{Z}_{N}$. There arises the question as to whether there exist particular sets of collocation parameters leading to a discrete convergence order (on $\bar{Z}_{N}$ ) that is higher than the global order.

In the subsequent analysis, the integrals

$$
J_{k}:=\int_{0}^{1} s^{k} \prod_{j=1}^{m}\left(s-c_{j}\right) d s, \quad k \in \mathbb{N},
$$

play a critical role.

THEOREM 3.1. Suppose that the given functions $q, p_{i}$, and $k_{i}(i=0,1)$ in the VIDE (1.1) are $m+p$ times continuously differentiable on their respective domains $I$ and $S$ $(d+1<p \leq m$ and $d \in\{0,1,2\})$, and assume that the collocation parameters $\left\{c_{j}\right\}_{j=1, \ldots, m}$ are chosen so that $J_{k}=0$ for $k=0,1, \ldots, p-1$, and $J_{p} \neq 0$. Then, for all quasiuniform mesh sequences $\left\{\prod_{N}\right\}$ with sufficiently small $h>0$, the following hold:

(i) if $u \in S_{m+d}^{(d)}\left(Z_{N}\right)$ is the approximate solution defined by the exact collocation equation (1.8) and $y$ is the exact solution of (1.1), then

$$
\max _{t_{n} \in \bar{Z}_{N}}\left|y\left(t_{n}\right)-u\left(t_{n}\right)\right|=O\left(h^{m+p}\right), \quad \text { as } h \longrightarrow 0, N h \leq \gamma T,
$$

(ii) if the quadrature formulas (1.12) satisfy (2.3) and (2.4), the quadrature formulas (1.13) satisfy (2.5) and (2.6), and $\hat{u} \in S_{m+d}^{(d)}\left(Z_{N}\right)$ is the approximate solution defined by the discretized collocation equation (1.16), then

$$
\max _{t_{n} \in \bar{Z}_{N}}\left|y\left(t_{n}\right)-\hat{u}\left(t_{n}\right)\right|=O\left(h^{\alpha}\right), \quad \text { as } h \longrightarrow 0, N h \leq \gamma T,
$$

where $\alpha=\min \left\{m+p, s_{0}\right\}$ and $s_{0}=\min \left\{r_{0}+1, r_{1}\right\}+1$.

Proof. (i) The exact collocation equation (1.8) can be written in the form

$$
\begin{aligned}
u^{\prime \prime}(t)= & q(t)+p_{0}(t) u(t)+p_{1}(t) u^{\prime}(t)-\delta(t) \\
& +\int_{0}^{t} k_{0}(t, s) u(s) d s+\int_{0}^{t} k_{1}(t, s) u^{\prime}(s) d s,
\end{aligned}
$$

where $\delta(t)$ (the residual) is (at least) continuous on each of the subintervals $\sigma_{n}$ and vanishes at the collocation points $X(N), t \in I$. Then, by (3.4) and (1.1), the error function 
satisfies

$$
\begin{aligned}
e^{\prime \prime}(t)= & p_{0}(t) e(t)+p_{1}(t) e^{\prime}(t)+\delta(t) \\
& +\int_{0}^{t} k_{0}(t, s) e(s) d s+\int_{0}^{t} k_{1}(t, s) e^{\prime}(s) d s,
\end{aligned}
$$

with initial conditions $e(0)=0, e^{\prime}(0)=0$.

The solution of (3.5) for $t=t_{n} \in \bar{Z}_{N}$ can be expressed in the form (see [4, pages 130-131])

$$
e\left(t_{n}\right)=h \sum_{i=0}^{n-1} E^{n, i},
$$

where $E^{n, i}$ is the quadrature error which, by (3.1), has an order $m+p$.

Hence,

$$
e\left(t_{n}\right)=\left|y\left(t_{n}\right)-u\left(t_{n}\right)\right|=O\left(h^{m+p}\right),
$$

which proves the first assertion of the theorem.

(ii) The second assertion of Theorem 3.1 now follows from (2.7) and (3.2).

It is well known that the orthogonality conditions $J_{k}=0(k=0, \ldots, p-1)$ imply that the degree of precision of the $m$-point interpolatory quadrature formula on $[0,1]$ based on the abscissas $\left\{c_{j}\right\}_{j=1, \ldots, m}$ is $m+p-1$. Since this degree of precision cannot exceed the value $2 m-1$, we always have $p \leq m$. Moreover, since the local order is required to be greater than or equal to the global order $(m+d+1)$, it is necessary that $d+2 \leq p$. The following corollary deals with some important special cases and its proof relies on the above theorem.

COROLLARY 3.2. Let the assumptions of Theorem 3.1 hold, then

(i) if the collocation parameters $\left\{c_{j}\right\}_{j=1, \ldots, m}$ are the Gauss(-Legendre) points for $(0,1)$, that is, the zeros of the shifted Legendre polynomial $P_{m}(2 s-1)$, then $J_{k}=0$ for $k=0, \ldots, m-1$, with $J_{m} \neq 0$, and hence

$$
\max _{t_{n} \in \bar{Z}_{N}}\left|y\left(t_{n}\right)-u\left(t_{n}\right)\right|=O\left(h^{2 m}\right), \quad \text { as } h \longrightarrow 0, N h \leq \gamma T,
$$

(ii) if the collocation parameters $\left\{c_{j}\right\}_{j=1, \ldots, m}$ are the Radau II points for $(0,1]$, that is, the zeros of the polynomial $P_{m-1}(2 s-1)-P_{m}(2 s-1)$, then $J_{k}=0$ for $k=$ $0, \ldots, m-2$, with $J_{m-1} \neq 0$, and hence

$$
\max _{t_{n} \in \bar{Z}_{N}}\left|y\left(t_{n}\right)-u\left(t_{n}\right)\right|=O\left(h^{2 m-1}\right), \quad \text { as } h \longrightarrow 0, N h \leq \gamma T .
$$

4. Numerical example. The convergence results derived in the preceding sections will be illustrated by applying various collocation methods to the following problem.

EXAMPLE 4.1. Consider the following integrodifferential equation of second order:

$$
y^{\prime \prime}(t)=q(t)+y(t)+\int_{0}^{t} t s y(s) d s, \quad y(0)=1, y^{\prime}(0)=1, t \in[0,1],
$$


TABLE 4.1. Approximate error when $m=2$ and $\left(\left\{c_{1}=1 / 2, c_{2}=1\right\}\right)$ (uniformly distributed collocation parameters).

\begin{tabular}{c|ccc}
\hline$d$ & $e_{1}$ & $e_{N / 2}$ & $e_{N}$ \\
\hline 0 & $1.68 \times 10^{-7}$ & 0.593657978 & 1.089198840 \\
1 & 0 & 0.000304350 & 0.012944677 \\
2 & 0 & 0.000303703 & 0.012941467 \\
3 & 0 & 0.000303661 & 0.012940922 \\
\hline
\end{tabular}

TABLE 4.2. Approximate error when $m=3$ and $\left(\left\{c_{1}=1 / 3, c_{2}=2 / 3, c_{3}=1\right\}\right)$ (uniformly distributed collocation parameters).

\begin{tabular}{c|ccc}
\hline$d$ & $e_{1}$ & $e_{N / 2}$ & $e_{N}$ \\
\hline 0 & $2.45 \times 10^{-5}$ & 0.015164638 & 0.172113704 \\
1 & 0 & 0.000303699 & 0.012941460 \\
2 & 0 & 0.000303701 & 0.012941459 \\
3 & 0 & 0.000303688 & 0.012941212 \\
\hline
\end{tabular}

TABLE 4.3. Approximate error when $m=2$ and (Radau II parameters) $\left(\left\{c_{1}=\right.\right.$ $\left.\left.1 / 3, c_{2}=1\right\}\right)$.

\begin{tabular}{c|ccc}
\hline$d$ & $e_{1}$ & $e_{N / 2}$ & $e_{N}$ \\
\hline 0 & $1.68 \times 10^{-7}$ & 0.593423722 & 1.089594757 \\
1 & 0 & 0.000304350 & 0.012944677 \\
2 & 0 & 0.000303704 & 0.012941470 \\
3 & 0 & 17489.97760 & $1.97 \times 10^{19}$ \\
\hline
\end{tabular}

TABLE 4.4. Approximate error when $m=3$ and (Radau II parameters) ( $\left\{c_{1}=\right.$ $\left.\left.(4-\sqrt{6}) / 10, c_{2}=(4+\sqrt{6}) / 10, c_{3}=1\right\}\right)$.

\begin{tabular}{c|ccc}
\hline$d$ & $e_{1}$ & $e_{N / 2}$ & $e_{N}$ \\
\hline 0 & 0.000027239 & 0.57170808 & 0.126648551 \\
1 & 0 & 0.0003033700 & 0.012941460 \\
2 & 0 & 0.000303703 & 0.012941464 \\
3 & 0 & $1.89 \times 10^{10}$ & $1.36 \times 10^{34}$ \\
\hline
\end{tabular}

with $q(t)$ chosen in such a way that it has the following exact solution:

$$
y(t)=t^{2}+e^{t} .
$$

Following Tables 4.1, 4.2, 4.3, and 4.4 illustrate the error approximations as

$$
\begin{aligned}
e_{1} & :=|y(0.01)-u(0.01)|, \\
e_{N / 2} & :=|y(0.5)-u(0.5)|, \\
e_{N} & :=|y(1)-u(1)|,
\end{aligned}
$$


where $u \in S_{m+d}^{d}\left(Z_{N}\right)$ is the approximate solution if $t_{i}:=i h \in Z_{N}$ with $h=0.01$ when $m \in\{2,3\}$ and $d \in\{0,1,2,3\}$.

As we can see from the above tables, the collocation spline method yields very good approximations when $d=1,2$. However, for $d=0$, the method performs poorly. But for the case $d=3$, the method converges if the collocation parameters are uniformly distributed and diverges if the collocation parameters are Radau II points, which can be seen from Tables 4.1, 4.2, 4.3, and 4.4.

\section{REFERENCES}

[1] H. Brunner, A survey of recent advances in the numerical treatment of Volterra integral and integro-differential equations, J. Comput. Appl. Math. 8 (1982), no. 3, 213-229.

[2] _ Implicit Runge-Kutta methods of optimal order for Volterra integro-differential equations, Math. Comp. 42 (1984), no. 165, 95-109.

[3] _ Polynomial spline collocation methods for Volterra integrodifferential equations with weakly singular kernels, IMA J. Numer. Anal. 6 (1986), no. 2, 221-239.

[4] - The approximate solution of initial-value problems for general Volterra integrodifferential equations, Computing 40 (1988), no. 2, 125-137.

[5] H. Brunner and J. D. Lambert, Stability of numerical methods for Volterra integrodifferential equations, Computing 12 (1974), no. 1, 75-89.

[6] H. Brunner, A. Pedas, and G. Vainikko, Piecewise polynomial collocation methods for linear Volterra integro-differential equations with weakly singular kernels, SIAM J. Numer. Anal. 39 (2001), no. 3, 957-982.

[7] H. Brunner and P. J. van der Houwen, The Numerical Solution of Volterra Equations, CWI Monographs, vol. 3, North-Holland Publishing, Amsterdam, 1986.

[8] T. A. Burton, Volterra Integral and Differential Equations, Mathematics in Science and Engineering, vol. 167, Academic Press, Florida, 1983.

[9] I. Danciu, Polynomial spline collocation methods for Volterra integro-differential equations, Rev. Anal. Numér. Théor. Approx. 25 (1996), no. 1-2, 77-91.

[10] M. Miculá and G. Micula, Sur la résolution numérique des équations intégrales du type de Volterra de seconde espèce à l'aide des fonctions splines, Studia Univ. Babeş-Bolyai Ser. Math.-Mech. 18 (1973), no. 2, 65-68 (French).

[11] V. Volterra, Theory of Functionals and of Integral and Integro-Differential Equations, Dover Publications, New York, 1959, with a preface by G. C. Evans, a biography of Vito Volterra and a bibliography of his published works by E. Whittaker.

Edris Rawashdeh: Department of Mathematics, Central Michigan University, Mount Pleasant, MI 48859, USA

E-mail address: edi rash@yahoo.com

David McDowell: Department of Mathematics, Central Michigan University, Mount Pleasant, MI 48859, USA

E-mail address: mcdow1d@cmich.edu

Leela Rakesh: Department of Mathematics, Central Michigan University, Mount Pleasant, MI 48859, USA

E-mail address: 1 rakesh@aol.com 


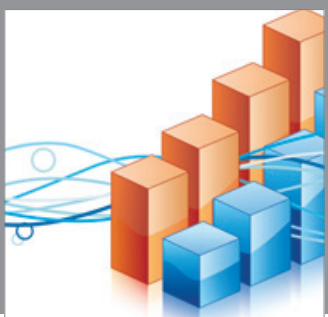

Advances in

Operations Research

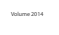

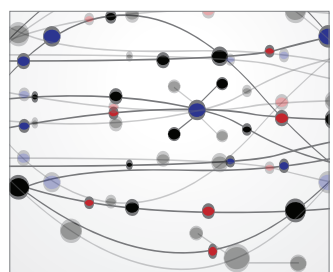

\section{The Scientific} World Journal
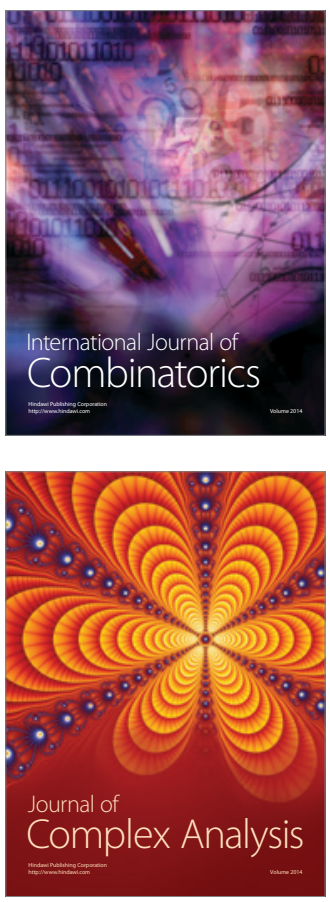

International Journal of

Mathematics and

Mathematical

Sciences
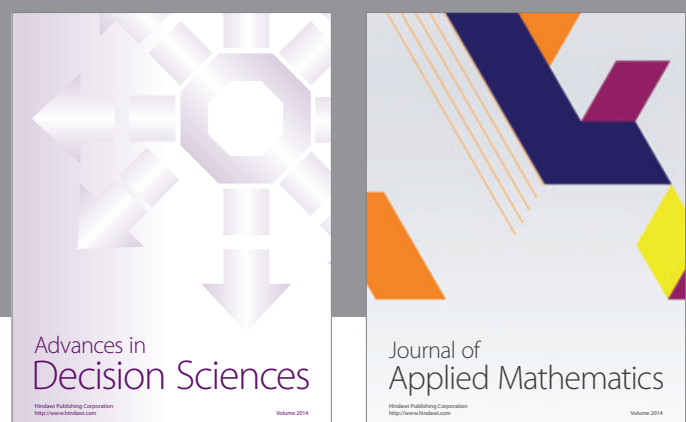

Journal of

Applied Mathematics
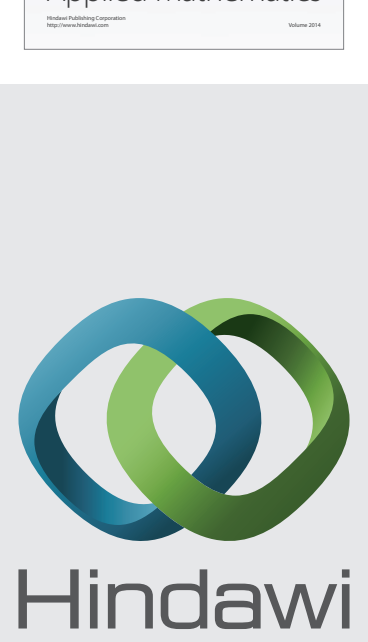

Submit your manuscripts at http://www.hindawi.com
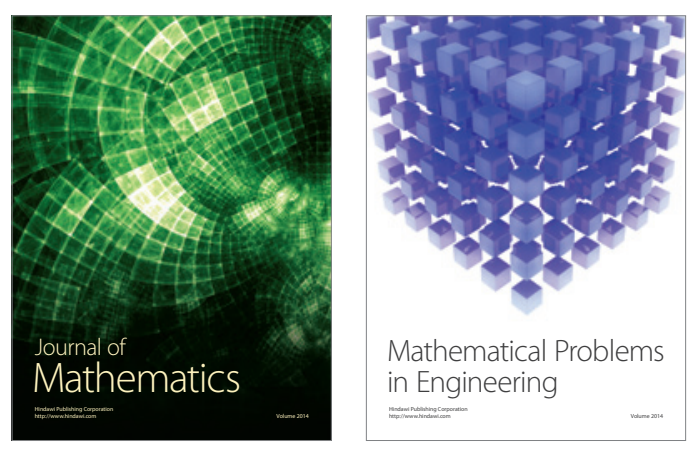

Mathematical Problems in Engineering
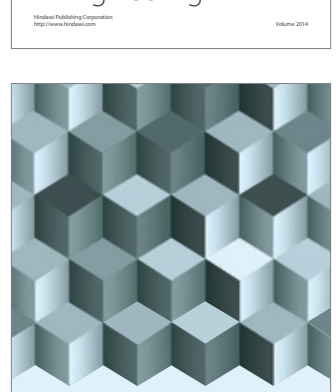

Journal of

Function Spaces
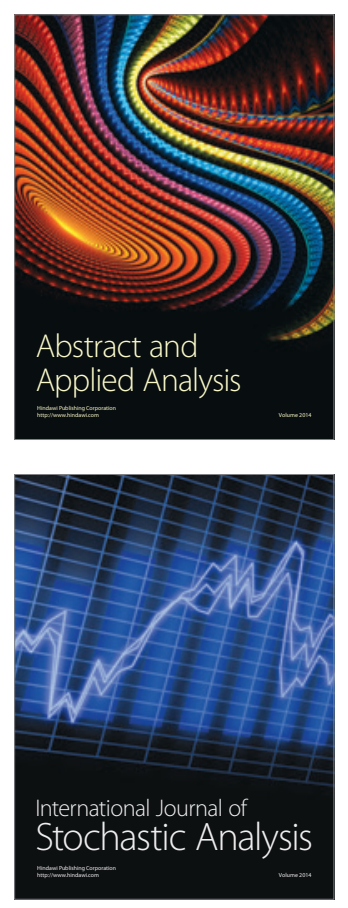

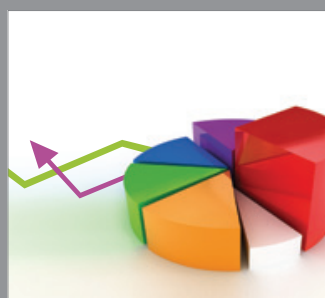

ournal of

Probability and Statistics

Promensencen
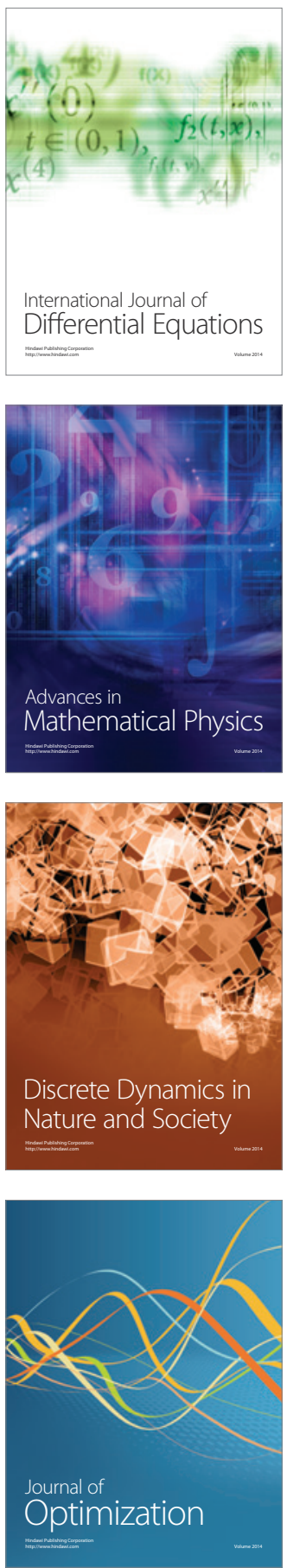